\title{
Berücksichtigung der Beschäftigten im Prozess der Digitalisierung aus Sicht von Personalverantwortlichen
}

\author{
Katja Stamer ${ }^{1,2}$ \\ Online publiziert: 5. November 2020 \\ (c) Der/die Autor(en) 2020
}

\section{Zusammenfassung}

Dieser Beitrag untersucht, inwiefern die Beschäftigten im Prozess der Digitalisierung aus der Perspektive von Personalverantwortlichen berücksichtigt werden. In diesem Kontext werden neben den seit Jahrzehnten thematisierten psychischen Beanspruchungen im Arbeitsumfeld zusätzlich die durch die Digitalisierung induzierten Stressoren eingeschätzt. Darüber hinaus wird überprüft, welche präventiven Maßnahmen in den Unternehmen eingesetzt werden, um die Stressoren für die Beschäftigten zu minimieren. Auf Basis eines querschnittlichen Untersuchungsdesigns und einer Stichprobe von Personalverantwortlichen $(N=241)$ aus Unternehmen verschiedener Branchen und Größen werden deskriptive Analysen durchgeführt.

Die Ergebnisse der Studie zeigen, dass in den Unternehmen, welche die Beschäftigten im Digitalisierungsprozess nicht ausreichend berücksichtigen, eine Zunahme an psychischen Krankheiten zu verzeichnen ist. Fehlt in den Unternehmen Kommunikation, Information und Transparenz, nehmen psychische Krankheiten ebenso zu. Weitere Studienergebnisse sind: Die Hälfte der Unternehmen (50,6\%) führt keine psychische Gefährdungsbeurteilung durch, ein Viertel (27\%) der Unternehmen setzt keine präventiven Maßnahmen zur Stressreduzierung ein.

Praktische Relevanz Die Zunahme von psychischen Krankheiten bei Beschäftigten und die daraus resultierenden Fehltage stehen teilweise in Verbindung mit den durch die Digitalisierung induzierten neuen Arbeitsbedingungen. Durch die Corona-Pandemie hat sich das Arbeitsumfeld durch den Einsatz von digitalen Medien und Prozessen kurzfristig verändert - die Digitalisierung wurde beschleunigt. Aus arbeitswissenschaftlicher Sicht ist es von großer Bedeutung, dass die Personalverantwortlichen die durch die Digitalisierung induzierten Stressoren wahrnehmen und entsprechende unterstützende Maßnahmen einleiten, um die Gesundheit der Beschäftigten zu erhalten.

Schlüsselwörter Digitalisierung · Psychische Stressoren · Präventive Maßnahmen · Messbarkeit präventiver Maßnahmen $\cdot$ Führungsverhalten

Prof. Dr. Katja Stamer

k.stamer@hb.dhbw-stuttgart.de

1 Florianstraße 15, 72160 Horb, Deutschland

2 Duale Hochschule Baden-Württemberg (DHBW), Stuttgart, Deutschland 


\title{
Consideration of employees in the process of digitization from the perspective of HR managers
}

\begin{abstract}
This contribution examines the consideration of employees in the process of digitization from the perspective of HR managers. In this context, in addition to the psychological stresses in the work environment, which have been a topic for decades, the stressors induced by digitization are also assessed and checked to see which preventive measures risk assessment, are used in the company to reduce the stressors for employees minimize. Descriptive analyzes are carried out on the basis of a cross-sectional study design and a sample of HR managers $(N=241)$ from companies of various industries and sizes. The results of the study show that companies that do not adequately consider employees in the digitization process have found an increase in mental illnesses. 27\% of the companies do not use preventive measures to reduce stress; $50.6 \%$ do not apply a psychological risk assessment. The lack of communication, information and transparency is also linked to the increase in mental illness in companies.

Practical relevance The increase in mental illnesses among employees and the resulting absence days are partly related to the new working conditions induced by digitalization. Due to the corona pandemic, the work environment changed at short notice through the use of digital media and processes-digitization was accelerated. From an ergonomic point of view, it is of great importance that the HR managers perceive the stressors induced by digitization and initiate appropriate supportive measures in order to maintain the health of the employees.
\end{abstract}

Keywords Digitalization - Psychological stressors - Preventive measures - Measurability of preventive methods · Leadership behaviour

\section{Einführung und Ziel der Untersuchung}

Laut einer Studie der Bertelsmann Stiftung und der Universität Witten/Herdecke (2020) ist die Digitalisierung für $60 \%$ der Führungskräfte ein wichtiges strategisches Thema. Der Prozess der Digitalisierung bringt allerdings neben technischen Herausforderungen auch Auswirkungen auf das Arbeitsumfeld der Beschäftigten mit sich (Arnold et al. 2016). Die Personalverantwortlichen der qualitativen Interview Studie IPA (2017) schätzen ein, dass für die Führungskräfte im digitalen Arbeitsumfeld neue Herausforderungen entstehen und sie die Beschäftigten im ChangeManagement begleiten müssen (Majkovic et al. 2018). Gemäß dem DGB-Index 2016 geben 82\% der befragten Beschäftigten an, dass ihre Arbeit durch die Digitalisierung beeinflusst wird. Die Mitarbeitenden sehen zwar in der digitalen Arbeitswelt Chancen und Vorteile - bessere Erreichbarkeit und Flexibilisierung der Arbeit - verspüren aber auch eine höhere Arbeitsmenge und weniger Einfluss auf das Tätigkeitsfeld sowie verschärfte Kontrolle und zunehmenden Arbeitsdruck (DGB 2017). Ragu-Nathan et al. (2008) sprechen in diesem Zusammenhang vom Phänomen des Technostresses. Gemäß dem BKK-Gesundheitsreport 2018 haben sich die durch psychische Krankheiten ausgelösten Fehltage in Deutschland in den vergangenen 40 Jahren mehr als verfünffacht (BKK 2018). Die daraus resultierenden wirtschaftlichen Ausfallkosten stiegen innerhalb von zehn Jahren von 12,4 Mrd auf 33,9 Mrd Euro an (Dpa 2019). Autoren des Handbuchs zur betrieblichen Gesundheitsförderung aus dem Jahr 1998 sprachen damals bereits von den seit Jahrzehnten verstärkten arbeitsbeding- ten psychischen Belastungen und Erkrankungen (Bamberg et al. 1998). Mit dem Faktor Digitalisierung, der die Arbeitsbedingungen bereits in den vergangenen Jahren stark verändert hat, beschleunigt zusätzlich die Corona-Pandemie laut der Bertelsmann-Zukunftsstudie (2020) die digitale Transformation der betrieblichen Arbeitswelt. Dabei lassen sich signifikante Zusammenhänge zwischen der Digitalisierung und der emotionalen Erschöpfung sowie Konflikten zwischen Arbeit und Familie erkennen (Böhm et al. 2016). $60 \%$ der deutschen Arbeitnehmer/innen erleben laut einer Studie des Deutschen Gewerkschaftsbunds (DGB) das Gefühl ,sehr oft oder oft bei der Arbeit gehetzt und unter Zeitdruck" zu sein (DGB 2017). Eine weitere Folge der Digitalisierung ist die zunehmende Erreichbarkeit der Mitarbeitenden außerhalb des Arbeitsumfelds. Beschäftigte, die erreichbarer sind, haben signifikant eher Probleme sich zu erholen als diejenigen, die das Privatleben und die Arbeit abgrenzen können (Rau und Göllner 2017).

Schwerpunktmäßig stellen verschiedene Studien - u. a. von Böhm et al. (2016) und des DGBs (2017) - vor allem die Herausforderungen in der digitalen Arbeitswelt aus Sicht der Beschäftigten dar. Jedoch werden in den Studien nicht die Wahrnehmung und Einschätzungen durch die Personalverantwortlichen betrachtet. Dieser arbeitswissenschaftliche Ansatz ist allerdings relevant, da sowohl die Führungskräfte und als auch die Mitarbeitenden aus der Personalabteilung u.a. für die Gesundheit der Beschäftigten verantwortlich sind (DGUV 2014). Im Mittelpunkt dieses Beitrags werden deshalb u. a. die psychischen Stressfaktoren auf die Beschäftigten - induziert durch die Digitalisierung - aus der Perspektive von Personalverantwort- 
lichen (Mitarbeitenden der Personalabteilung, Geschäfts-, Abteilungs- bzw. Teamleitung, Betriebsrat und Fachkraft für Arbeitssicherheit) eingeschätzt. Außerdem wird untersucht, welche präventive Maßnahmen aus dem betrieblichen Gesundheitsmanagement und welches Führungsverhalten aktuell wahrgenommen wird, um psychische Krankheiten bzw. Stressoren in digitalen Arbeitsumfeld zu minimieren. Des Weiteren wird ermittelt, ob diese Maßnahmen aus der Sicht der Befragten messbar sind und welchen Einfluss der Einsatz präventiver Methoden auf den Krankenstand im Hinblick auf psychische Krankheiten hat.

\section{Konzeptionelle Grundlagen und Vorüberlegungen}

Traum et al. (2017) stellt bei der Untersuchung von Definitionen zur Digitalisierung fest, dass bei den meisten Definitionen das von dieser Entwicklung betroffene Individuum fehlt. Die Digitalisierung hat jedoch starken Einfluss auf kognitive und motivationale Prozesse bei den Beschäftigten (Antoni und Ellwart 2017) und führt möglicherweise zu negativen gesundheitlichen Folgen (VBG 2015). Die fehlende Integration des Individuums in die Definition der Digitalisierung ist laut Traum et al. (2017) folgenschwer für das Verständnis der Auswirkungen auf die Wirtschaft, in welcher das Handeln der Beschäftigten entscheidend ist.

\subsection{Psychische Stressoren, induziert durch die Digitalisierung}

Stressoren, hervorgerufen durch die Digitalisierung, wie beispielsweise neue Formen der Mensch-Maschine-ArbeitInteraktion, gestiegene Qualifikationsanforderungen, eine veränderte Arbeitsorganisation, verstärkte Kontrolle und weniger Autonomie sowie zunehmende Beschleunigung in der Arbeitswelt und vermehrter Arbeitsdruck lassen sich gemäß der qualitativen Interviewstudie von Diebig et al. (2017) u.a. mit dem Job-Demand-Control-Modell von Karasek (1979) in Verbindung bringen. Diese Faktoren können eine Einschränkung im Handlungsspielraum und eine Erhöhung der Arbeitsanforderungen darstellen. Werden der Handlungsspielraum und die Autonomie reduziert, kann dies zur Minderung der Motivation bei den Beschäftigten (Hackman und Oldham 1975) und letztlich auch zu negativen gesundheitlichen Folgen führen (Diebig et al. 2017). Des Weiteren entsteht durch eine Überforderung aufgrund fehlender Qualifikation, aber auch durch zu häufige Qualifikationsmaßnahmen (vor allem für ältere Beschäftigte) (Lübke 2015; forsa main 2016) sowie durch zunehmende Beschleunigung und Druck im Arbeitsumfeld Stress (Csikszentmihalyi 1975). Darüber hinaus rufen neue Formen der Mensch-Maschine-Arbeit-Interaktion (Mensch und Maschine interagieren statt nebeneinander her) sowie geänderte Arbeitsorganisationen im Rahmen der Digitalisierung ein Unsicherheitserleben mit der Angst vor einem Arbeitsplatzverlust hervor (BAuA 2017). Durch die erwartete abnehmende interpersonale Kommunikation (Diebig et al. 2017) sowie durch eine zunehmende Abwesenheit der Führungskraft wegen der Flexibilisierung der Arbeit (Rau und Göllner 2017) kann es zu einer Reduzierung der Motivation aufgrund mangelnder Anerkennung und fehlender Bestätigung kommen (Hackman und Oldham 1975). Expertinnen und Experten in der qualitativen Interviewstudie von Diebig et al. (2017) sprechen außerdem von einer zunehmenden Entgrenzung der Arbeit durch die ständige Erreichbarkeit der Beschäftigten sowie durch die zunehmende Flexibilisierung der Arbeitszeit und des Arbeitsorts (Diebig et al. 2017). Diese Flexibilisierung stellt zwar mehr Freiheit und Selbstentfaltung dar, birgt aber das Risiko der Selbstüberforderung und einer sozialen Entbindung mit der Gefahr von Schlafstörungen, Erholungsunfähigkeit sowie weiteren stress-assoziierten Krankheiten (Rau und Göllner 2017, Gimpel et al. 2018).

\subsection{Psychische Gefährdungsbeurteilung}

Eine Möglichkeit, Stressoren zu erfassen und zu bewerten bietet die psychische Gefährdungsbeurteilung (GBU), die seit 2013 im Arbeitsschutz verankert ist (DGUV 2014). Studien zeigen, dass Unternehmen Umsetzungskonflikte bei der Durchführung der psychischen GBU haben (BAuA 2014; Lenhardt und Beck 2016; Leitao und Greiner 2017) und nur 66,2\% der Unternehmen eine psychische GBU durchführen (EU-OSHA 2016) Konflikte entstehen gemäß Wulf et al. (2017) u.a. durch die unklare Interpretation und Bewertung von Zielen und Ergebnissen sowie durch die jeweilige Wahrnehmung der GBU durch die beteiligten Interessensvertreter/innen.

\subsection{Betriebliches Gesundheitsmanagement}

Eine Auswertung von Studien durch die iga (2019) zeigt, dass sich betriebliches Gesundheitsmanagement (BGM) positiv auf die Gesundheit der Mitarbeitenden auswirkt und Fehlzeiten um rund ein Viertel sinken, wenn Unternehmen Angebote zur Verbesserung der körperlichen und der psychischen Verfassung der Beschäftigten fördern (Pieper et al. 2015). Gleichwohl scheint das BGM gemäß der Trendstudie von Prümper et al. (2014) vor allem bei kleinen und mittelgroßen Unternehmen noch kein Schwerpunktthema zu sein. Die Trendstudie zeigt, dass Unternehmen vor allem verhältnisbezogene Maßnahmen - wie die ergonomische Gestaltung der Arbeitsumgebung, flexible Arbeitszeitmodelle oder Maßnahmen zur Vereinbarkeit von Privatleben und Beruf - einsetzen. Verhaltensbezogene Maßnahmen - 
wie zum Beispiel Angebote zur Stressbewältigung und zur gesunden Ernährung - werden seltener eingesetzt (Prümper et al. 2014).

\subsection{Führungsverhalten im Kontext der Digitalisierung}

Geänderte Rahmenbedingungen im digitalen Arbeitsumfeld sind eine Herausforderung für die Beschäftigten. Sie können unterschiedliche Ängste aufgrund empfundener Bedrohung eines möglichen Arbeitsplatzverlustes oder Konflikte (Vereinbarkeit Beruf und Privatleben) hervorrufen (Lazarus 1999). Die von Gregersen et al. (2011) gesichteten Studien und Metaanalysen zeigen, dass transformale Führung mit einer verstärkten Orientierung für die Beschäftigten, intensiver Kommunikation und einer guten Beziehung zum Vorgesetzten (Böhm et al. 2016) eine gesundheitsförderliche Wirkung auf die Beschäftigten hat und Stress, Burnout und Fehlzeiten reduziert.

\subsection{Messbarkeit und ökonomischer Nutzen von BGM}

Um die Unternehmen vom Einsatz gesundheitsförderlicher Maßnahmen zu überzeugen, sollte ein gesundheitlicher und wirtschaftlicher Mehrwert nachweisbar sein. Gemäß dem iga-Report 28 (2015) können sich durch das BGM in Unternehmen die Kennzahlen Produktivität und Kosten sowie das Arbeitsklima und die Arbeitszufriedenheit positiv entwickeln (Pfannstiel und Mehlich 2016). Andere Untersuchungen sagen dagegen, dass es im Gesundheitsbereich relativ wenig aussagekräftige Kennzahlen gibt, die für die Messbarkeit des BGMs geeignet sind.

\section{Untersuchungsziele und Forschungsfragen}

Eines der Hauptziele dieser Studie ist es, den aktuellen Status quo zu erfassen: Inwiefern werden die Beschäftigten im Prozess der Digitalisierung aus Sicht von Personalverantwortlichen im Unternehmen berücksichtigt. Die Befragung beinhaltet außerdem die Einschätzung von Personalverantwortlichen, ob psychische Erkrankungen im Unternehmen zunehmen. Des Weiteren wird die Wahrnehmung der Auswirkungen von psychischen Stressoren - induziert durch die Digitalisierung (Diebig et al. 2017) - erfasst. Weitere Untersuchungsgegenstände sind Einschätzungen zur Durchführung von psychischen GBUs, zu präventiven Maßnahmen im Rahmen des BGM, zur Messbarkeit dieser Maßnahmen sowie zur Beurteilung des Führungsverhaltens, das Beschäftigte im geänderten Arbeitsumfeld begleitet.
Folgende Forschungsfragen lassen sich aus den genannten Zielen ableiten:

1. Berücksichtigung der Beschäftigten (F1)

In welchem Maße werden die Beschäftigten aus Sicht von Personalverantwortlichen im Rahmen des Digitalisierungsprozesses im Unternehmen berücksichtigt?

Kann ein Zusammenhang zwischen dem aktuellen Stand der Digitalisierung der Produkte bzw. der Prozesse und der Berücksichtigung der Mitarbeitenden festgestellt werden?

2. Psychische Krankheiten und Stressoren, induziert durch die Digitalisierung (F2)

Wie schätzen die befragten Personalverantwortlichen die Zunahme der psychischen Krankheiten im Unternehmen und die durch die Digitalisierung induzierten psychischen Stressoren ein?

3. Präventive Maßnahmen zur Stressreduzierung in den Unternehmen (F3)

Führen Unternehmen eine psychische Gefährdungsbeurteilung durch?

Welche präventiven Methoden aus dem BGM werden eingesetzt?

Wie wird das Führungsverhalten, um die Mitarbeitenden in dem durch die Digitalisierung geänderten Arbeitsumfeld zu begleiten, wahrgenommen?

4. Unterschiede zwischen den Einschätzungen je nach Aufgabenfeld der Befragten (F4)

Gibt es innerhalb der Stichprobe, abhängig von der Position in Unternehmen, Unterschiede bei der Einschätzung

a) inwiefern Mitarbeitende im Prozess der Digitalisierung berücksichtigt werden

b) wie weit der Einfluss von psychischen Stressoren durch die Digitalisierung reicht

c) wie die Begleitung der Beschäftigten im geänderten Arbeitsumfeld durch entsprechendes Führungsverhalten erfolgt und inwiefern innerhalb der vergangenen zwei Jahre eine psychische GBU durchgeführt wurde,

d) ob eine Zunahme von psychischen Krankheiten feststellbar ist.

5. Messbarkeit präventiver Maßnahmen (F5)

Sind die dargestellten präventiven Maßnahmen messbar?

\subsection{Methode und Operationalisierung}

Um die Relevanz der Thematik vorab zu prüfen, wurde ein Pre-Test mit zehn strukturierten qualitativen Experteninterviews in Personalabteilungen bei mittleren bis groBen Unternehmen der verarbeitenden Industrie durchgeführt. Die Datenerhebung für den Pre-Test erfolgte telefonisch im Juni 2019 mithilfe eines halbstrukturierten Interviewleitfadens. Im nächsten Schritt wurde ein Online- 
Fragebogen gemäß dem Fragebogenprozess von Bortz und Döring (2006) mit Einfach- und Mehrfachwahlfragten, Rankingskalen (Lienert und Raatz 1998) sowie einer offenen Fragestellung erstellt. Im Skalenniveau der Items wurden nominal- und ordinalskalierte Variablen eingesetzt (Zöfel 2003). Der Online-Fragebogen wurde im Zeitraum Juli bis August 2019 an die Personalverantwortlichen von Kooperationsunternehmen der Fakultät Technik der DHBW verschickt. Die Rücklaufquote betrug $15 \%(n=241)$. Die Auswertung der quantitativen Daten erfolgte durch deskriptive Verfahren (Zöfel 2003) sowie durch für die bei den vorherrschenden Skalenniveaus in Frage kommenden Tests mit SPSS. Die Antworten der offenen Frage wurden in Anlehnung an Mayring (2002) mithilfe einer induktiven Kategorienentwicklung ausgewertet.

\subsection{Stichprobe}

Die Stichprobe $(n=241)$ setzt sich zusammen aus Verantwortlichen der Personalabteilungen und dem BGM $(n=92)$, aus Vertreterinnen und Vertreter der Geschäftsführung ( $n=39)$, und aus Abteilungs- oder Teamleitung $(n=100)$ sowie aus Beschäftigen des Bereichs Arbeitssicherheit und dem Betriebsrat $(n=10)$.

Die Personalverantwortlichen aus der Stichprobe sind bei Unternehmen folgender Branchen und Unternehmensgrößen beschäftigt (Tab. 1 und 2).

Tab. 1 Branchen der Stichprobe

Table 1 Sectors of the sample

\begin{tabular}{ll}
\hline Branche & In \% \\
\hline Industrie und Maschinenbau & 60,6 \\
Automobil- und Fahrzeugbau & 11,6 \\
IT-Technologie & 10,0 \\
Technische und Finanzdienstleistungen & 6,2 \\
Bauwesen und Architektur & 3,7 \\
Elektrotechnik & 2,9 \\
Medizintechnik & 0,8 \\
Beratung und Consulting & 0,8 \\
Transport und Logistik & 0,4 \\
Andere Branchen & 2,9 \\
\hline
\end{tabular}

$n=241$

Tab. 2 Unternehmensgrößen der Stichprobe

Table 2 Enterprise sizes in the sample

\begin{tabular}{ll}
\hline Anzahl Beschäftigte im Unternehmen & In \% \\
\hline Weniger als 10 & 2,5 \\
Zwischen 10 und 50 & 6,6 \\
Zwischen 51 und 250 & 26,6 \\
Zwischen 215 und 1000 & 34,0 \\
Mehr als 1000 & 30,3 \\
\hline
\end{tabular}

$n=241$

\section{3 Überprüfung der Gütekriterien und Datenqualität}

Vor der Versendung des Online-Fragebogens an die Unternehmen wurde mit zwölf ausgesuchten Experten und Expertinnen ein Pre-Test durchgeführt, um die Validität und Objektivität zu prüfen (Lienert und Raatz 1998). Die Überprüfung der Reliabilität der acht Items ,,psychische Stressoren" basierend auf der qualitativen Untersuchung von Diebig et al. (2017) sowie ,Führungsverhalten im Kontext der Digitalisierung“ (acht Items, entnommen und angelehnt an den MLQ Fragebogen (Felfe und Goihl 2014)) wurde mittels einer Konsistenzanalyse, durch die Berechnung des Werts von Cronbach Alpha durchgeführt. Ein akzeptabler Wert von 0,75 wurde für die Items der psychischen Stressoren und ein guter Wert von 0,85 für die Items zum Führungsverhalten ermittelt (Backhaus et al. 2011).

\section{Deskription der Ergebnisse und Beantwortung der Fragestellungen}

\subsection{Allgemeine Informationen zum aktuellen Stand der Digitalisierung in den Unternehmen}

Zunächst wurde in der Online-Befragung ermittelt, ob es im Unternehmen eine Strategie zur Digitalisierung gibt und inwiefern die Digitalisierung in den Bereichen Prozesse und Produkte vorangeschritten ist. 67,6\% der befragten Unternehmen verfügen über ein Konzept bzw. eine Strategie zum Thema Digitalisierung, 24,5\% haben kein Konzept und $7,9 \%$ der Befragten wissen nicht, ob ein Konzept existiert. Die befragten Personen geben zum aktuellen Stand der Digitalisierung der Prozesse auf einer Likert-Skala von 1 (gar nicht entwickelt) bis 5 (sehr weit entwickelt) einen Mittelwert $(M)$ von 3,07 mit einer Standardabweichung $(S D)$ von 0,844 an. Zum aktuellen Stand der Digitalisierung von Produkten weist das Ergebnis der Befragung einen Mittelwert von 2,64 mit einer Standardabweichung von 1,306 auf.

Gemäß den Aussagen der Stichprobe sind vor allem folgende Abteilungen von der Umstellung auf die Digitalisierung betroffen (Nennungen in \% $(n=241))$ : IT $(53,3 \%)$, Forschung und Entwicklung (38,5\%), Produktion $(36 \%)$, Verwaltung allgemein $(30,2 \%)$, Vertrieb und Marketing $(25,4 \%)$, Logistik $(25,4 \%)$, Einkauf und Beschaffung $(23,1 \%)$.

Im nachfolgenden Absatz werden die Forschungsfragen anhand entsprechender deskriptiver Analysen beantwortet. 


\subsection{Berücksichtigung der Beschäftigten im Rahmen der Digitalisierung (F1)}

Das Ergebnis zur Frage, wie sehr Mitarbeitende in der Digitalisierungsstrategie berücksichtigt werden" stellt sich wie folgt dar: $14,9 \%$ der Befragten $(n=36)$,gar nicht“, 49,8\% (n=120) ,teilweise“, 27,8\% (n=67) ,gut“ und $7,5 \%(n=18)$,,sehr gut“ (wobei ,gar nicht“ auf der LikertSkala mit einer 0 , ,teilweise" $=1$, ,gut" $=2$ und ,,sehr gut" mit einer 3 festgelegt wurde, $M=1,28, S D=0,807$ ).

Beim Vergleich der verschiedenen Unternehmensgrößen und der Branchen lässt sich kein signifikanter Unterschied bei den Ergebnissen zur Berücksichtigung der Beschäftigten in der Digitalisierungsstrategie feststellen.

Zwischen dem aktuellen Stand der Digitalisierung für Produkte sowie Prozesse und der Einschätzung der „Berücksichtigung der Beschäftigten im Digitalisierungsprozess" besteht kein Zusammenhang. Das bedeutet, dass ein Unternehmen, welches einen höheren Entwicklungsstand in der Digitalisierung von Produkten und Prozessen ausweist, nicht gleichzeitig die Mitarbeitenden im Bereich der Digitalisierung eher berücksichtigt als Unternehmen, die einen niedrigeren Entwicklungsstand im Rahmen der Digitalisierung bestätigen.

Allerdings gibt es einen Zusammenhang zwischen dem Maß an Berücksichtigung der Beschäftigten und der Zunahme an psychischen Krankheiten im Unternehmen $(n=177$, $\left.\mathrm{X}^{2}(3)=14.626, p=0,002\right)$. Das bedeutet, je weniger die Beschäftigten berücksichtigt werden, desto eher wird eine Zunahme an psychischen Krankheiten festgestellt.

\subsection{Aktueller Stand zur Einschätzung psychischer Krankheiten im Unternehmen sowie den durch die Digitalisierung induzierten psychischen Stressoren (F2)}

34,4\% der befragten Personalverantwortlichen stellen keine Zunahme der psychischen Krankheiten im Unternehmen fest. $39 \%$ berichten von einer Zunahme und 26,6\% können diese Frage nicht beantworten. Bei diesem Ergebnis lassen sich keine Unterschiede innerhalb der Branchen feststellen, jedoch zwischen den Unternehmensgrößen $\left(\mathrm{X}^{2}\right.$ (4) $=34.282, p=0,00, n=177)$. Die Personalverantwortlichen größerer Unternehmen stellen eher eine Zunahme an psychischen Krankheiten fest als die Personalverantwortlichen von kleineren Unternehmen.

Die Einschätzung der psychischen Stressoren, die für die Gesundheit der Beschäftigten im Prozess der Digitalisierung eine negative Auswirkung haben können (Diebig et al. 2017), wird in Tab. 3 durch den Mittelwert und die Standardabweichung je Stressor dargestellt:

Tab. 3 zeigt, dass die psychischen Stressoren ,zunehmende Beschleunigung der Arbeitswelt und vermehrter Arbeits-
Tab. 3 Mittelwert $(M)$ und Standardabweichung $(S D)$ der psychischen Stressoren, induziert durch die Digitalisierung

Table 3 Mean value (M) and standard deviation (SD) of mental stressors induced by digitalisation

\begin{tabular}{lll}
\hline Item & $M$ & $S D$ \\
\hline Neue-Formen der Mensch-Maschine-Arbeit & 2,69 & 1,143 \\
Zunehmende Beschleunigung der Arbeitswelt & 3,88 & 1,092 \\
und vermehrter Arbeitsdruck & & \\
Entgrenzung der Arbeit und Freizeit & 3,05 & 1,168 \\
Gestiegene Qualifikationsforderungen & 3,59 & 1,054 \\
Veränderte Arbeitsorganisation & 3,60 & 1,218 \\
Verstärkte Kontrolle & 2,96 & 1,259 \\
Einschränkung der Autonomie & 2,66 & 1,238 \\
Weniger direktes Feedback durch Vorgesetzte & 2,78 & 1,225 \\
\hline
\end{tabular}

$n=241,1=$ kein Einfluss, $2=$ kaum Einfluss, $3=$ Einfluss, $4=$ hoher Einfluss, $5=$ sehr hoher Einfluss

druck“ mit $M=3,88$, ,die veränderte Arbeitsorganisation“ mit $M=3,6$, die ,gestiegenen Qualifikationsanforderungen“" mit $M=3,59$ und ,die Entgrenzung der Arbeit und Freizeit“ mit $\mathrm{M}=3,05$ die höchsten Mittelwerte aufweisen. Somit hat die Digitalisierung aus Sicht von Personalverantwortlichen auf diese genannten psychischen Stressoren einen Einfluss bzw. eine Tendenz zu hohem Einfluss.

Bei der Einschätzung der Stressoren gibt es keine signifikanten Unterschiede zwischen den Branchen. Bei der Gegenüberstellung der Unternehmensgrößen lässt sich aber bei den Stressoren „gestiegene Qualifikation“ ( $\mathrm{X}^{2}$ $(20)=40.006, p=0,005, n=241)$, bei ,veränderte Arbeitsorganisation" $\left(\mathrm{X}^{2}(20)=32.261, p=0,041, n=241\right)$ sowie „Einschränkung der Autonomie“ $\left(\mathrm{X}^{2}(20)=43.664\right.$, $p=0,002, n=241)$ ein signifikanter Unterschied feststellen. Mit Zunahme der Unternehmensgröße haben gemäß der Stichprobe diese drei psychischen Stressoren einen höheren Einfluss als bei kleineren Unternehmen.

Die in Tab. 4 dargestellten Items werden von den Personalverantwortlichen, die eine Zunahme an psychischen Krankheiten im Unternehmen feststellen, signifikant höher

Tab. 4 Einschätzung der psychischen Stressoren in Verbindung mit der Zunahme psychischer Krankheiten (nur Darstellung von Signifikanzen durch den Chi-Quadrat Test)

Table 4 Assessment of mental stressors in connection with the increase in mental illness (only the Chi-square test significances are shown)

\begin{tabular}{llll}
\hline & Wert & $d f$ & $p$ \\
\hline $\begin{array}{l}\text { Neue Formen Mensch-Maschine- } \\
\begin{array}{l}\text { Arbeit } \\
\text { Beschleunigung Arbeitswelt und } \\
\text { vermehrter Arbeitsdruck }\end{array}\end{array}$ & 11.900 & 5 & $0,036^{*}$ \\
Entgrenzung Arbeit und Freizeit & 11.130 & 5 & $0,049^{*}$ \\
\hline$n=177$ & & & \\
$* p<0,05, * * p<0,01$ & & &
\end{tabular}


wahrgenommen als von denjenigen, die keine Zunahme an psychischen Krankheiten identifizieren.

\subsection{Präventive Maßnahmen zur Stressreduzierung (F3)}

GBU In Bezug auf die Durchführung einer psychischen $G B U$ geben $50,6 \%$ der Personalverantwortlichen an, dass innerhalb der vergangenen zwei Jahren keine GBU durchgeführt worden sei. Bei 34,4\% der Unternehmen wurde in den vergangenen zwei Jahren im Unternehmen eine psychische GPU angewendet, 14,9\% können dazu keine Angaben machen. Dabei gibt es signifikante Unterschiede zwischen den Unternehmensgrößen $\left(X^{2}(8)=16.324, p=0,038\right.$, $n=241)$ : Größere Unternehmen führten innerhalb der vergangenen zwei Jahren eher eine GBU durch als kleinere Unternehmen. Zwischen den Branchen sind bezüglich des Einsatzes einer GBU keine Unterschiede festzustellen.

Es lässt sich kein Zusammenhang zwischen einer durchgeführten psychischen GBU und deren Einfluss auf die Wahrnehmung, ob psychische Krankheiten zugenommen haben, feststellen.

Methoden aus dem betrieblichen Gesundheitsmanagement Die durch eine induktive Kategorienentwicklung codierten Antworten der offenen Frage bezüglich der eingesetzten Präventionsmaßnahmen zeigt in Tab. 5 folgende Kategorien (es werden nur die Methoden mit mehr als fünf Nennungen gelistet).

Führungsverhalten - zur Begleitung der Beschäftigten im Prozess der Digitalisierung In Bezug auf die Einschät-

Tab. 5 Art und Anzahl der Nennungen von präventiven Maßnahmen Table 5 Type and number of mentions of preventive measures

\begin{tabular}{ll}
\hline Art der präventiven Methode & $\begin{array}{l}\text { Anzahl Nen- } \\
\text { nungen }\end{array}$ \\
\hline Keine präventiven Methoden vorhanden & 65 \\
Schulungen und Präventionsangebote & 48 \\
Persönlicher Austausch und Gespräche & 46 \\
Angebote aus dem BGM & 37 \\
Arbeitszeitmodelle & 27 \\
Vorsorge und Untersuchungen (inkl. interner und & 19 \\
externer psychischer Betreuung) & \\
Führungskräfteschulung & 17 \\
Gefährdungsbeurteilungen & 12 \\
Flexible Arbeitsplätze (Home Office) & 9 \\
Sportangebote & 7 \\
Befragungen der Mitarbeitenden & 6 \\
Coaching & 6 \\
Gutes Arbeitsklima schaffen & 5 \\
Gesundheitstage & 5 \\
\hline
\end{tabular}

$n=241$
Tab. 6 Mittelwerte $(M)$ und Standardabweichungen $(S D)$ für die Wahrnehmung des Führungsverhaltens im Kontext der Digitalisierung Table 6 Mean values (M) and standard deviations (SD) for the perception of leadership behaviour in the context of digitisation

\begin{tabular}{lll}
\hline Item & $M$ & $S D$ \\
\hline Intensive Kommunikation über Veränderungen & 3,23 & 1,212 \\
Verstärkte Information und Transparenz & 3,22 & 1,151 \\
Unterstützung bei Qualifikationsmaßnahmen & 3,33 & 1,117 \\
Abgrenzung von Arbeit und Privatleben festle- & 2,75 & 1,138 \\
gen und selbst vorleben & & \\
Coaching und Unterstützung bei Konflikten & 3,20 & 1,201 \\
Verstärktes Feedback für die Mitarbeitenden & 3,03 & 1,178 \\
\hline
\end{tabular}

$n=241,1=$ darauf wird kein Wert, bzw. $5=$ darauf wird sehr großer Wert gelegt

Tab. 7 Darstellung der Items des Führungsverhaltens, die signifikant in Verbindung mit der Zunahme psychischer Krankheiten stehen Table 7 Presentation of the items of leadership behaviour that are significantly associated with the increase in mental illness

\begin{tabular}{llll}
\hline & Wert & Df & $p$ \\
\hline $\begin{array}{l}\text { Intensive Kommunikation über } \\
\text { Veränderungen }\end{array}$ & 11.907 & 5 & $0,036^{*}$ \\
$\begin{array}{l}\text { Verstärkte Information und Trans- } \\
\text { parenz }\end{array}$ & 17.679 & 5 & $0,003^{* *}$ \\
\hline$n=177$ & & & \\
$* p<0,05, * * p<0,01$ & & &
\end{tabular}

zung der Skala Führungsverhalten ergeben sich innerhalb der Skala von $1=$,Es wird kein Wert darauf gelegt“ bis $5=$,Auf dieses Führungsverhalten wird sehr großer Wert gelegt“, folgende in Tab. 6 dargestellte Mittelwerte bzw. Standardabweichungen.

Zwischen den Unternehmensgrößen und den Branchen lässt sich kein Unterschied bei der Wahrnehmung des Führungsverhaltens, die Beschäftigten im Digitalisierungsprozess zu begleiten, feststellen.

Die Darstellung in Tab. 7 zeigt die Items aus der Skala „Führungsverhalten, um die Mitarbeitenden im neuen Arbeitsumfeld zu begleiten“, die in Zusammenhang mit der Einschätzung der Zunahme von psychischen Krankheiten im Unternehmen stehen.

Die Personalverantwortlichen, die eine Zunahme an psychischen Krankheiten feststellen, schätzen die Items „Intensive Kommunikation über Veränderungen“ und ,verstärkte Information und Transparenz" signifikant mit einem niedrigen Mittelwert im Vergleich zu denjenigen, die keine $\mathrm{Zu}$ nahme von psychischen Krankheiten identifizieren.

\subsection{Unterschiede der Einschätzung innerhalb der Stichprobe je nach Aufgabenfeld der Befragten (F4)}

Da die Stichprobe aus Personalverantwortlichen aus verschiedenen Positionen (Mitarbeitende aus Personalabtei- 
lung, BGM, Ausbildung, Geschäftsführung, Abteilungsund Teamleitung, sowie Betriebsrat und Fachkraft für Arbeitssicherheit) besteht, gilt es nun zu überprüfen, ob es innerhalb dieser Stichprobe unterschiedliche Einschätzungen zu den Fragestellungen gibt.

Bei einem Vergleich der Beurteilung, inwiefern die Beschäftigen in der Digitalisierungsstrategie berücksichtigt werden, zeigen sich signifikante Unterschiede $\left(\chi^{2}(15)=\right.$ $36.788, p=0,001, n=241)$ je nach Aufgabengebiet der Stichprobe im Unternehmen. Während die Geschäftsleitung die Berücksichtigung der Beschäftigten im Rahmen der Digitalisierung als tendenziell eher gut bis sehr gut einschätzt $(M=1,79$, basierend auf der Likert-Skala $0=$ gar nicht, $1=$ teilweise, $2=$ gut, $3=$ sehr gut), sprechen die anderen Befragten (Abteilungs- und Teamleitung $(M=1,10)$, Mitarbeitende der Personalabteilung/BGM/Ausbildung $(M=1,29))$, Betriebsrat/Fachkraft für Arbeitssicherheit $(M=0,90)$, eher von einer „teilweisen“" Berücksichtigung.

Bei einem Vergleich der Aussagen, ob eine Zunahme an psychischen Krankheiten festzustellen ist, zeigen sich zwischen den Positionen ebenso signifikante Unterschiede $(p<0,01)$. Beschäftige in der Personalabteilung, in Ausbildung und im BGM sowie aus der Arbeitssicherheit und dem Betriebsrat sprechen eher von einer Zunahme an psychischen Krankheiten als die Geschäftsführung.

Zur Einschätzung der psychischen Stressoren lässt sich kein signifikanter Unterschied zwischen den verschiedenen Positionen von Personalverantwortlichen feststellen. Generell zeigt sich jedoch beim Vergleich aller Mittelwerte, dass die Geschäftsleitung die psychischen Stressoren niedriger einschätzt als die Akteure der anderen Positionen.

Bezüglich der Frage, ob in den vergangenen zwei Jahren eine Gefährdungsbeurteilung durchgeführt wurde, zeigen sich zwischen den Aussagen je nach Position signifikante Unterschiede $\left(\chi^{2}(10)=25.571, p=0,004, n=241\right)$. Auffällig ist, dass vor allem die Abteilungs- und Teamleitungen diese Aussage eher verneinen als die Befragten in den anderen Positionen.

Die Geschäftsleitung nimmt grundsätzlich das Führungsverhalten zur Unterstützung der Beschäftigten im Rahmen der Digitalisierung bei allen Items höher wahr, als die anderen Personalverantwortlichen $(1=$ darauf wird kein Wert, bis $5=$ darauf wird sehr großer Wert gelegt). Die Mittelwerte für die Items zum Führungsverhalten zeigen - wie in Tab. 8 dargestellt - bei den vier Items „Intensive Kommunikation über Veränderungen“, „Verstärkte Information und Transparenz“, „Coaching und Unterstützung bei Konflikten“ sowie „Verstärktes Feedback für die Mitarbeitenden" signifikante Unterschiede je nachdem welche Befragten die Einschätzung abgegeben haben.

Die Geschäftsleitung schätzt das Führungsverhalten bei den in Tab. 8 dargestellten Kriterien signifikant höher ein als die anderen Personalverantwortlichen diese Kriterien beurteilen.

\subsection{Messbarkeit präventiver Maßnahmen (F5)}

Bei der Frage, ob sich der Erfolg von eingesetzten Maßnahmen zur Stressprävention in betriebswirtschaftlichen Kennzahlen messen lässt, sagen $12 \%(n=29)$ der Befragten „nein“ 18,7\% (n=45) der Befragten ,ja“. 69,3\% ( $n=167)$ beurteilen den Erfolg der Maßnahmen als schwer messbar. Bei einem Vergleich zwischen den Unternehmensgrößen und Branchen, sowie zwischen den unterschiedlichen Positionen im Unternehmen zeigen sich keine signifikanten Unterschiede hinsichtlich dieser Aussagen.

\section{Diskussion, Fazit und Limitationen}

Die Studie wurde durchgeführt, weil es in den vergangenen zehn Jahren (2007 bis 2017) (BAuA 2018; Gimbel et al. 2018) zu einer starken Zunahme an psychischen Krankheiten aufgrund von digitalem Stress bei Beschäftigten und daraus resultierenden Fehltagen gekommen ist - und untersucht die Thematik aus Sicht von Personalverantwortlichen. Durch die Corona-Pandemie - seit der viele Beschäftigte im Homeoffice arbeiten, sich kurzfristig auf digitale Medien einlassen mussten, die Entgrenzung von Arbeit und Freizeit schwieriger wird - gewinnt die Thematik zusätzlich an erheblicher Relevanz. In dieser Untersuchung liegt der Schwerpunkt auf der Einschätzung der Personalverantwortlichen: Inwiefern berücksichtigen sie die Beschäftigten im Rahmen der Digitalisierung?
Tab. 8 Mittelwerte mit signifikanten Unterschieden je nach Position bei der Wahrnehmung von vier Items aus der Skala des Führungsverhaltens

Table 8 Mean values with significant differences according to position in the perception of four items from the scale of leadership behaviour

\begin{tabular}{lllll}
\hline PositionItem & $\begin{array}{l}\text { Kommunikation } \\
\text { M }\end{array}$ & $\begin{array}{l}\text { Information/ } \\
\text { Transparenz } \\
\text { M }\end{array}$ & $\begin{array}{l}\text { Coaching } \\
\text { M }\end{array}$ & $\begin{array}{l}\text { Feedback } \\
\text { M }\end{array}$ \\
\hline $\begin{array}{l}\text { Geschäftsleitung } \\
\text { Mitarbeitende Personalabteilung der }\end{array}$ & 3,62 & 3,67 & 3,64 & 3,51 \\
Ausbildung und BGM & 3,12 & 3,12 & 3,16 & 2,97 \\
Abteilungs- und Teamleitung & 3,25 & & & \\
Arbeitssicherheit/Betriebsrat & 2,50 & 3,23 & 3,13 & 2,97 \\
\hline
\end{tabular}

$n=241,1=$ darauf wird kein Wert, bzw. $5=$ darauf wird sehr großer Wert gelegt $* p<0,05$ 
Die dargestellten Ergebnisse zeigen, dass die Unternehmen der Stichprobe in Bezug auf den aktuellen Stand der Digitalisierung von Prozessen und Produkten gut entwickelt sind. Jedoch werden die Beschäftigten innerhalb dieser Strategie nur teilweise berücksichtigt. Mit erheblichen Folgen: Bei Unternehmen, welche die Mitarbeitenden weniger im Prozess der Digitalisierung einschließen bzw. begleiten, lässt sich eine stärkere Zunahme von psychischen Krankheiten feststellen als bei Unternehmen, welche die Beschäftigten berücksichtigen. Bei den von Diebig et al. (2017) dargestellten psychischen Stressfaktoren, induziert durch die Digitalisierung haben - gemäß den Ergebnissen der vorliegenden Studie - die folgenden Faktoren aus Sicht von Personalverantwortlichen den größten Einfluss auf die Gesundheit der Beschäftigten: die zunehmende Beschleunigung der Arbeitswelt und der vermehrte Arbeitsdruck, eine veränderte Arbeitsorganisation, gestiegene Qualifikationsanforderungen sowie die „Entgrenzung der Arbeit und Freizeit“. Die „zunehmende Beschleunigung der Arbeitswelt und vermehrter Arbeitsdruck" sowie die „Entgrenzung der Arbeit und Freizeit" stehen außerdem im Zusammenhang mit einer Zunahme von psychischen Krankheiten. Dieses Ergebnis deckt sich mit den in der Einleitung genannten Resultaten aus Studien von Rau und Göllner (2017), des DGB (2017) sowie Dettmers (2017) und Reimann (2019).

Um die Beschäftigten im Digitalisierungsprozess zu begleiten und psychische Stressoren zu reduzieren, werden präventive Maßnahmen wie die psychische GBU, Angebote aus dem BGM oder direktes unterstützendes Führungsverhalten eingesetzt. Die vorliegende Studie zeigt, dass nur $34,4 \%$ der befragten Personalverantwortlichen in Ihrem Unternehmen in den vergangenen zwei Jahren eine psychische GBU durchgeführt haben. Wie in Studien aus früheren Jahren (Uhle und Treier 2015; Ahlers 2015) erarbeitet, zeigt somit auch das Ergebnis dieser Untersuchung, dass die psychische GBU bis heute in vielen Unternehmen nicht flächendeckend durchgeführt wird (Wulf et al. 2017). Bei der vorliegenden Analyse wird außerdem sichtbar, dass vor allem Abteilungs- und Teamleitungen im Vergleich zu den Befragten in anderen Positionen die Durchführung einer GBU innerhalb der vergangenen zwei Jahre eher verneinen oder darüber nicht informiert sind. Somit spielt die interne Kommunikation und entsprechende Transparenz ebenfalls eine zentrale Rolle im Rahmen dieser Thematik. Das Ergebnis dieser Untersuchung zeigt, dass signifikant eher größere Unternehmen als kleinere eine psychische GBU durchführen. Ahlers (2015) identifiziert Probleme bei der Umsetzung durch unübersichtliche Messinstrumente und Tools sowie mangelnde nachhaltige Maßnahmenkonzepte nach der Durchführung und die Unterlassung der GBU aufgrund zu hoher Kosten. Pfannstiel und Mehlich (2016) zeigen auf, dass vor allem kleinere und mittelgroße Unternehmen aufgrund fehlender ärztlicher und sicherheits- technischer Betreuung, Schwierigkeiten haben, die GBU durchzuführen. Nach Ahlers (2015) fühlen sich die für die GBU verantwortlichen Personen nicht umfassend geschult und überfordert, die GBU effizient durchzuführen. Einen Blick zu den europäischen Nachbarn Dänemark und Frankreich zu werfen (Ahlers 2015; Uhle und Treier 2015) gibt Unternehmen Hilfestellung, klarere Regeln und Konzepte zur GBU festzulegen. Denn beide Länder verfügen über eindeutige Regularien zur effizienten Durchführung einer GBU (Janetzke und Ertel 2016).

Die Ergebnisse der Untersuchung zeigen eine Vielfalt an aktuell eingesetzten präventiven Maßnahmen u.a. aus dem BGM. $27 \%$ der Befragten berichten allerdings, dass im Unternehmen keine Maßnahmen zur Stressreduzierung eingesetzt werden. Knapp 70\% der Befragten schätzen die Messbarkeit von präventiven Methoden aus dem BGM als schwierig (12\% sogar als gar nicht messbar) ein. Dieses Ergebnis spiegelt ähnliche Aussagen aus dem iga-Report 40 (2019) wider. Allerdings zeigen zahlreiche Studien (Aldana 2001; Chapman 2012; Palletier 2005 und 2009 in Uhle und Treier 2015), dass sich die Investition in ein BGM aus betriebswirtschaftlicher Sicht lohnt. Neben subjektiven und qualitativen Auswirkungen - wie höherer intrinsischer Motivation und größerer Zufriedenheit der Mitarbeitenden - geben Fehlzeitenquoten, Krankheitsquoten und die Fluktuation als Kennzahlen im Unternehmen Auskunft über den Erfolg präventiver Maßnahmen (Uhle und Treier 2015). Die Verwendung einer Health Balanced Scorecard, angepasst auf das jeweilige Unternehmen, ist eine weitere Methode zur Darstellung des Erfolgs des BGMs (Wellmann 2012).

Nach Uhle und Treier (2015) spielen Führung und Kommunikation maßgeblich eine große Rolle bei der Prävention von psychischen Krankheiten. Die Ergebnisse der Studie zeigen, dass eine niedrige Einschätzung der ,intensiven Kommunikation“ sowie der „Information und Transparenz" signifikant in Zusammenhang mit der Zunahme an psychischen Krankheiten steht. Das Führungsverhalten zur „Abgrenzung von Arbeit und Privatleben festlegen und selbst vorleben" wird am niedrigsten wahrgenommen, obwohl dieses Kriterium signifikant mit der Zunahme von psychischen Krankheiten im Zusammenhang steht. Da die Kommunikation, Information und Transparenz wichtige Ansatzpunkte im BGM darstellen (Uhle und Treier 2015), und dadurch psychische Stressoren reduziert werden können (DGUV 2014; BAuA 2017), ist es von Bedeutung, dass Führungskräfte sich über die Bedeutung dieser gesundheitsförderlichen Maßnahmen (Uhle und Treier 2015) bewusst sind und in ihr Führungsverhalten integrieren. Auch das „,verstärkte Feedback für die Beschäftigten“ wird in diesem Beitrag nur als „mittelmäßig“ wahrgenommen, obgleich Untersuchungen (DGUV 2014; Gregersen et al. 2011) die Bedeutung von Anerkennung und Feedback im Führungsverhalten herausstellen. 
Die Ergebnisse zeigen einen Unterschied auf, je nachdem die Merkmale zum Führungsverhalten von der Geschäftsleitung oder von anderen Personalverantwortlichen aus der Stichprobe eingeschätzt wurden. Bei allen sechs Items zur Beurteilung des Führungsverhaltens zeigen die Ergebnisse, dass die Geschäftsleitung „,mehr Wert“ darauf legt - im Vergleich zur Wahrnehmung dieser Kriterien durch die anderen Befragten. Bei den Items ,intensive Kommunikation“, ,verstärkte Transparenz“, „Coaching/ Unterstützung bei Konflikten“ sowie ,verstärktes Feedback" weichen die Wahrnehmungen durch die Geschäftsleitung und die anderen Befragten signifikant voneinander ab. Somit liegen hier unterschiedliche Selbsteinschätzungen vor, welche mit dem Self-Serving-Bias (Harris und Schaubroeck 1988) begründet werden können. Sie spiegeln sich in der geringen Übereinstimmung der Geschäftsleitung und den anderen Beschäftigten aus der Personalabteilung, den Abteilungen und Teams sowie aus dem Betriebsrat und der Arbeitssicherheit wider. Da es sich bei der Digitalisierung von Prozessen und Produkten um einen ChangeManagement-Prozess handelt, ist es von Bedeutung, die Beschäftigen als wichtige Akteure zu berücksichtigen. Das Scheitern von Veränderungsprozessen wird in der ChangeManagement-Literatur mit dem Fehlen einer Vision, der unzureichenden Vermittlung der Veränderungsnotwendigkeit in der Organisation, und der Nicht-Beachtung von Widerständen und Ängsten bei den Mitarbeitenden begründet (Burnes 2011; Kotter 2011). Deshalb gilt es, als Führungsverantwortliche/r diese Herausforderungen $\mathrm{zu}$ erkennen und entsprechend $\mathrm{zu}$ handeln. Allerdings zeigt sich, dass den Führungskräften oftmals das Wissen zur richtigen Umsetzung von Veränderungen ohne gesundheitsschädigende Auswirkungen für die Beschäftigten fehlt (Kieselbach et al., 2009). Die BAuA (2018) hat zu dieser Problematik das Projekt ,Entwicklung und Pilotierung von Qualifizierungstools für Führungskräfte und Personalverantwortliche zur Unterstützung erfolgreicher Restrukturierung (EPIQUA)“ durchgeführt und damit die Grundlage für die Konzeption einer betrieblichen Weiterbildungsmaßnahme für Führungskräfte und für den Betriebsrat zum Thema ,Restrukturierung und Gesundheit" geschaffen. Damit die Umsetzung eines Konzepts zur Digitalisierung gelingt, müssen Führungskräfte eine Vorbildfunktion übernehmen und die Beschäftigten im Change-Prozess begleiten (Paefgen-Laß 2018). Während der Corona-Pandemie, in der psychische Stressoren (Diebig et al. 2017) - wie veränderte Arbeitsorganisation durch Homeoffice und damit verbunden Entgrenzung der Arbeit und Freizeit sowie weniger Feedback durch Vorgesetzte - verstärkt Auswirkungen auf die psychische Gesundheit der Beschäftigen haben können, bekommt entsprechendes Führungsverhalten wie „Transparenz“, „Klarheit schaffen“, „,Kommunikation“, „Unterstützung bei Konflikten“, „Unterstützung bei Qualifikationsmaßnahmen im Umgang mit digitalen Medien“, zusätzlich erhebliche Relevanz (Kals 2020; Burger 2020; Ragu-Nathan et al. 2008).

Als Handlungsempfehlungen für die Personalverantwortlichen in den Unternehmen lässt sich zusammenfassen: Zunächst einmal überprüfen, ob die eigene Wahrnehmung bezüglich der neuen digitalen Arbeitsbedingungen mit denen der Beschäftigten übereinstimmt. Dazu sind entsprechende Gespräche und Beobachtungen wichtig (DGB 2017). Zum anderen sollte die psychische GBU mit definierten Prozessen zur Überprüfung von psychischen Stressoren - u.a. induziert durch die Digitalisierung - regelmäßig durchgeführt und daraus stressreduzierende Maßnahmen aus dem BGM abgeleitet werden. Dabei können u.a. wissenschaftlich fundierte und bereits in der Praxis erprobte Vorgehensweisen der Fokusgruppe „Gesunde Arbeit durch innovative Arbeitsformen und Assistenzsysteme“ (Kratzer 2019) angewendet werden. Ein weiteres Handlungsfeld in den Unternehmen ist die Weiterbildung von Führungskräften insbesondere zu den Themen „Kommunikation“ und „transparente Information“. Auch zu diesen Themen können Forschungsgruppen aus der genannten Fokusgruppe (Kratzer 2019) wissenschaftlich fundierte Empfehlungen vorlegen. Darüber hinaus führen Qualifizierungsprogramme und die technische Unterstützung für die Beschäftigten zur Reduzierung von Stress und Ängsten (Ragu-Nathan et al. 2008). Die Messbarkeit von BGM-Maßnahmen kristallisiert sich durch diese Arbeit als zukünftiges Forschungsthema heraus. Nur so können Unternehmen von einer Investition in das BGM und die damit verbundene Nachhaltigkeit überzeugt werden (igaReport 2015). Dabei ist u.a. die Wirksamkeit der Nutzung einer Health Balanced Scorecard zu überprüfen.

Trotz der relevanten Ergebnisse der Untersuchung sind Limitierungen zu nennen. Die Stichprobe wurde vor allem in der technisch orientierten Branche durchgeführt und ist wegen der Besonderheit des verwendeten Samples nicht repräsentativ. Deshalb wäre eine Ausweitung der Umfrage auf weitere Branchen und auf eine größere Stichprobe bei den Gruppen der Personalverantwortlichen - beispielweise auf die Arbeitssicherheit und Betriebsrat - interessant. Des Weiteren beschränkt sich die Studie ausschließlich auf deskriptive Analysen und Korrelationen. Hier ist bei den Aussagen der Befragten das Thema Subjektivität der individuellen Wahrnehmungen nicht auszuschließen. Interessant wäre es, diese Studie als Längsschnittstudie erneut durchzuführen, um festzustellen, ob mit weiterem technischem digitalem Fortschritt, auch die Beschäftigten in der Digitalisierungsstrategie stärkere Berücksichtigung finden als zum jetzigen Zeitpunkt.

Funding Open Access funding enabled and organized by Projekt DEAL. 
Open Access Dieser Artikel wird unter der Creative Commons Namensnennung 4.0 International Lizenz veröffentlicht, welche die Nutzung, Vervielfältigung, Bearbeitung, Verbreitung und Wiedergabe in jeglichem Medium und Format erlaubt, sofern Sie den/die ursprünglichen Autor(en) und die Quelle ordnungsgemäß nennen, einen Link zur Creative Commons Lizenz beifügen und angeben, ob Änderungen vorgenommen wurden.

Die in diesem Artikel enthaltenen Bilder und sonstiges Drittmaterial unterliegen ebenfalls der genannten Creative Commons Lizenz, sofern sich aus der Abbildungslegende nichts anderes ergibt. Sofern das betreffende Material nicht unter der genannten Creative Commons Lizenz steht und die betreffende Handlung nicht nach gesetzlichen Vorschriften erlaubt ist, ist für die oben aufgeführten Weiterverwendungen des Materials die Einwilligung des jeweiligen Rechteinhabers einzuholen.

Weitere Details zur Lizenz entnehmen Sie bitte der Lizenzinformation auf http://creativecommons.org/licenses/by/4.0/deed.de.

\section{Literatur}

Ahlers E (2015) Leistungsdruck, Arbeitsverdichtung und die (ungenutzte) Rolle von Gefährdungsbeurteilungen. WSI MITTEILUNGEN 3/2015, 194-201. https://www.boeckler.de/wsi-mitteilungen_ 53826_53844.htm. Zugegriffen: 30. Nov. 2019

Antoni $\mathrm{CH}$, Ellwart T (2017) Informationsüberlastung bei digitaler Zusammenarbeit Ursachen, Folgen und Interventionsmöglichkeiten. Gruppe. Interaktion. Organisation. Z Angew Organisationspsychol 48(2019):305-315. https://doi.org/10.1007/s11612-0170392-4

Arnold D, Arntz M, Gregory T, Steffens S, Zierahn U (2016) Herausforderungen der Digitalisierung für die Zukunft der Arbeitswelt. ZEW policybrief. Centre for European Economic Research (ZEW), Mannheim. https://madoc.bib.uni-mannheim.de/43782/ 1/pb16-08.pdf. Zugegriffen: 1. Dez. 2019

Backhaus K, Erichson B, Plinke W, Weber R (2011) Multivariate Analysemethoden, 13. Aufl. Springer, Heidelberg

Bamberg E, Ducki A, Metz AM (1998) Handbuch betriebliche Gesundheitsförderung. Arbeits-und organisationspsychologische Methoden und Konzepte. Hogrefe, Göttingen

BKK Gesundheitsreport (2018) https://www.bkk-dachverband.de/ publikationen/bkk-gesundheitsreport.html. Zugegriffen: 20. Dez. 2019

Böhm SA, Bourovoi K, Brzykcy AZ, Kreissner LM, Breier C (2016) Auswirkungen der Digitalisierung auf die Gesundheit von Berufstätigen: Eine bevölkerungsrepräsentative Studie in der Bundesrepublik Deutschland. Universität St. Gallen, St. Gallen

Bortz J, Döring N (2006) Forschungsmethoden und Evaluation: Für Human- und Sozialwissenschaftler, 4. Aufl. Springer, Berlin

Bundesanstalt für Arbeitsschutz und Arbeitsmedizin (BAuA) (2017) Psychische Gesundheit in der Arbeitswelt Arbeitsplatzunsicherheit (Job Insecurity) Forschung Projekt F 2353 B. Köper, S. Gerstenberg. Dortmund/Berlin/Dresden. https://www. baua.de/DE/Angebote/Publikationen/Berichte/F2353-2f.pdf? blob=publicationFile\&v=5. Zugegriffen: 25. Nov. 2019

Bundesanstalt für Arbeitsschutz und Arbeitsmedizin (BAuA) (2018) Qualifizierungstools für Führungskräfte und Betriebsräte bei betrieblichen Restrukturierungen. 1. Auflage, Dortmund/Berlin/ Dresden. https://www.baua.de/DE/Angebote/Publikationen/Be richte/F2340.pdf?_blob=publicationFile\&v=5. Zugegriffen: 29 . Nov. 2019

Bundesanstalt für Arbeitsschutz und Arbeitsmedizin (BAuA) (2014) Gefährdungsbeurteilungen psychischer Belastung - Erfahrungen und Empfehlungen. Erich Schmidt, Berlin

Burger C (2020) VDI Nachrichten 03.04.2020. Führung in Zeiten der Corona Krise. Bitcom: Homeoffice die Regel. https://www.vdi- nachrichten.com/karriere/fuehrung-in-zeiten-der-corona-krise/. Zugegriffen: 25. Juni 2020

Burnes B (2011) Introduction: Why does change fail, and what can we do about it? J Chang Manag 11(4):445-450

Csikszentmihalyi M (1975) Beyond boredom and anxiety. Jossey-Bass publishers San Francisco Washington, London. http://psy2.ucsd. edu/ nchristenfeld/Happiness_Readings_files/Class\%207\%20\%20Csikszentmihalyi\%201975.pdf. Zugegriffen: 25. Nov. 2019

Dettmers J (2017) How extended work availability impairs wellbeing - the role of detachment and work-family-conflict. J Work Stress 31:24-41. https://doi.org/10.1080/02678373.2017.1298164

DGUV (2014) Führung und psychische Gesundheit. https://www.dguv. de/medien/inhalt/praevention/fachbereiche_dguv/fb-gib/psyche/ broschuere_fuehrung.pdf. Zugegriffen: 24. Nov. 2019

DGB (2017) Verbreitung, Folgen und Gestaltungsaspekte der Digitalisierung in der Arbeitswelt. Auswertungsbericht auf Basis des DGB-Index Gute Arbeit 2016. Institut DGB-Index Gute Arbeit Berlin. https://index-gute-arbeit.dgb.de/++co++1c40dfc8-b95311e7-8dd1-52540088cada. Zugegriffen: 1. Dez. 2019

Diebig M, Müller A, Angerer P (2017) Psychische Belastungen in der Industrie 4.0 Eine selektive Literaturübersicht zu (neuartigen) Belastungsbereichen. Arbeitsmed Sozialmed Umweltmed 52(11):832-839

dpa (2019) Süddeutsche Zeitung 26.03.2019. Fehltage wegen psychischer Probleme stark angestiegen. dpa-Newskanal. https:// www.sueddeutsche.de/gesundheit/gesundheit-fehltage-wegenpsychischer-probleme-stark-angestiegen-dpa.urn-newsml-dpacom-20090101-190326-99-554783. Zugegriffen: 30. Nov. 2019

EU-OSHA-European Agency for Safety and Health at Work (2016) Second European Survey of enterprises on new and emerging risks (ESENER-2) Overview report:managing safety and health at work. https://osha.europa.eu/de/european-survey-enterprisesnew-and-emerging-risks-esener. Zugegriffen: 24. Nov. 2019

Felfe J, Goihl K (2014) Deutscher Multifactor Leadership Questionnaire (MLQ). Zusammenstellung sozialwissenschaftlicher Items und Skalen (ZIS) https://doi.org/10.6102/zis22

forsa main (2016) Umfrage: Wie steht es um den Wert der Weiterbildung? Haufe-Akademie. https://www.haufe-akademie.de/ perspektiven/wert-der-weiterbildung/. Zugegriffen: 2. Dez. 2019

Gimpel H, Lanzl J, Manner-Romberg T, Nüske N (2018) Digitaler Stress in Deutschland. Working-Papier Forschungsförderung, Nr. 101. Hans-Böckler-Stiftung, Düsseldorf.

Gregersen S, Kuhnert S, Zimber A, Nienhaus A (2011) Führungsverhalten und Gesundheit - Zum Stand der Forschung. Gesundheitswesen 73(1):3-12

Hackman R, Oldham GR (1975) Development of the job diagnostic survey. J Appl Psychol. https://doi.org/10.1037/H0076546

Harris MM, Schaubroeck J (1988) A meta-analysis of self-supervisor, self-peer, and peer-supervisor ratings. Pers Psychol 41:43-62

Iga-Report 28 (2015) Wirksamkeit und Nutzen arbeitsweltbezogener Gesundheitsförderung und Prävention Zusammenstellung der wissenschaftlichen Evidenz für den Zeitraum 2006 bis 2012. BKK, DV, DGUV, AOK-Bv, vdek, Berlin

Iga-Report 40 (2019) Wirksamkeit und Nutzen arbeitsweltbezogener Gesundheitsförderung und Prävention. usammenstellung der wissenschaftlichen Evidenz für den Zeitraum 2012 bis 2018. BKK, DV, DGUV, AOK-Bv, vdek. Berlin. https://www.igainfo.de/fileadmin/redakteur/Veroeffentlichungen/iga_Reporte/ iga-Report_40_Zusammenfassung_.pdf. Zugegriffen: 24. Nov. 2019

Janetzke H, Ertel M (2016) Gefährdungsbeurteilung psychosozialer Belastungen im europäischen Vergleich. Working Paper Forschungsförderung Nummer 016, Juni 2016. Hans-BöcklerStiftung, Düsseldorf. https://www.boeckler.de/pdf/p_fofoe_WP_ 016_2016.pdf. Zugegriffen: 24. Nov. 2019

Kals U (2020) Frankfurter Allgemeine 06.04.2020. Was sollen Führungskräfte tun? https://www.faz.net/aktuell/karriere-hochschule/ buero-co/was-sollen-fuehrungskraefte-in-der-corona-krise-tun- 
16714466.html?printPagedArticle=true\#pageIndex_2. Zugegriffen: 25. Juni 2020

Kasarek RA (1979) Job demands, job decision latitude, and mental strain. Implications for job design. Adm Sci Q 24:285-308. https://doi.org/10.2307/2392498

Kieselbach T, Armgarth E, Bagnara S, Elo AL, Jefferys S, Joling C, Widerszal-Bazyl M (2009) Health in restructuring. Innovative approaches and policy recommendations (HIRES). Mering, Hampp, München

Kotter JP (2011) Leading Change. Wie Sie Ihr Unternehmen in Acht Schritten erfolgreich verändern, 1. Aufl. Vahlen, München

Kratzer N (2019) Arbeit der Zukunft. Digital, multilokal, dynamisch. Fokusgruppe „Gesunde Arbeit durch innovative Arbeitsformen und Assistenzsysteme, München

Lazarus RS (1999) Stress and emotion: a new synthesis. Springer, New York

Leitao S, Greiner B (2017) Psychosocial, health promotion and safety culture management - are health and safety practitioners involved? Saf Sci 91:84-92

Lenhardt U, Beck D (2016) Prevalence and quality of workplace risk assessments-findings from a representative company survey in Germany. Federal Institute for Occupational Safety and Health Berlin, Germany, S. 48-56. https://www.baua.de/DE/Angebote/ Publikationen/Aufsaetze/artikel100.pdf?_blob=publicationFile \& v=3. Zugegriffen: 2. Dez. 2019

Lienert GA, Raatz U (1998) Testaufbau und Testanalyse, 6. Aufl. Beltz, Weinheim

Lübke F (2015) So finden Sie mit 50+ die richtige Weiterbildung, In die Welt. https://www.welt.de/wirtschaft/karriere/bildung/ article138646312/So-finden-Sie-mit-50-die-richtige-Weiterbil dung.html. Zugegriffen: 24. Nov. 2019

Majkovic AL, Werkmann-Karcher B, Gundrum E, Birrer J, Genner S, Probst L, Huber R, Pfister A (2018) IAP Studie 2017 - Teil 2. Der Mensch in der Arbeitswelt 4.0. Ergebnisse der qualitativen Interviews. IAP Institut für Angewandte Psychologie der ZHAW Zürcher Hochschule für Angewandte Wissenschaften, Zürich

Mayring P (2002) Qualitative Sozialforschung. Beltz, Weinheim

Paefgen-Laß M (2018) Digitalisierung ist Chefsache. Online-Artikel. Springer Professional. https://www.springerprofessional. de/change-management/unternehmenskultur/digitalisierung-istchefsache/15179940. Zugegriffen: 2. Dez. 2019

Pfannstiel MA, Mehlich H (2016) Betriebliches Gesundheitsmanagement. Konzepte, Maßnahmen, Evaluation. Springer Gabler, Wiesbaden, S 379
Pieper C, Schröer S, Haupt J, Kramiga I (2015) Wirksamkeit und Nutzen betrieblicher Prävention, Zusammenstellung der wissenschaftlichen Evidenz 2006-2012, in: iga-Report 28, BKK, DV, DGUV, AOK-Bv, vdek. Berlin. https://www.iga-info.de/ fileadmin/redakteur/Veroeffentlichungen/iga_Reporte/Dokumen te/iga-Report_28_Wirksamkeit_Nutzen_betrieblicher_Praeventi on.pdf. Zugegriffen: 2. Dez. 2019

Prümper J, Zinke J, Nachtwei J, Hornung S (2014) Das Ziel wird langsam deutlich. - Trendstudie Betriebliches Gesundheitsmanagement. Personalmagazin 04:60-62 (Spezial Gesundheitsmanagement)

Ragu-Nathan TS, Tarafdar M, Ragu-Nathan BS, Tu Q (2008) The consequences of technostress for end users in organizations: conceptual development and empirical validation. Inf Syst Res 19(4):417-433

Rau R, Göllner M (2017) Erreichbarkeit gestalten, oder doch besser die Arbeit? Z Arbeits Organisationspsychol 63(1):1-14

Reimann M (2019) Arbeitszeitgestaltung und psychische Gesundheit: Die vermittelnde Rolle von zeit- und belastungsbasierten Konflikten zwischen Berufs- und Privatleben. Z Arb Wiss. https://doi.org/ 10.1007/s41449-019-00171-0

Traum A, Müller C, Hummert H, Nerdinger FW (2017) Digitalisierung - Die Perspektive des arbeitenden Individuum. White Paper Series Nr. 1. Projekt-Kodima, Rostock

Uhle T, Treier M (2015) Betriebliches Gesundheitsmanagement. Gesundheitsförderung in der Arbeitswelt - Mitarbeitenden einbinden, Prozesse gestalten, Erfolge messen, 3. Aufl. Springer, Berlin Heidelberg, S 248-249

VBG (2015) Gefährdungsbeurteilung psychischer Belastungen. Handlungshilfe für die betriebliche Praxis. http://www.vbg.de/Shared Docs/Medien-Center/DE/Broschuere/Themen/Arbeitsschutz_or ganisieren/gefaehrdungsbeurteilung_psychischer_belastung.pdf? blob=publicationFile\&v=6. Zugegriffen: 24. Nov. 2019

Wellmann H (2012) Einsatz der Balanced Scorecard in der betrieblichen Gesundheitspolitik, 266 Arbeitspapier, Hans-Böckler-Stiftung. https://www.boeckler.de/pdf/p_arbp_266.pdf. Zugegriffen: 1. Dez. 2019

Wulf IC, Süß S, Diebig M (2017) Akteure der Gefährdungsbeurteilung psychischer Belastung - Perspektiven und Konflikte im betrieblichen Arbeits- und Gesundheitsschutz. Z Arb Wiss 71:296-304. https://doi.org/10.1007/s41449-017-0085-4

Zöfel P (2003) Statistik für Psychologen im Klartext. Pearson, München 